Preprint Version: 4th IEEE/RAS International Conference on Humanoid Robots, 2004., 2004, pp. $196-214$ Vol. 1

\title{
ADVANCED STEPS IN BIPED ROBOTICS: INNOVATIVE DESIGN AND INTUITIVE CONTROL THROUGH SPRING-DAMPER ACTUATOR
}

\author{
Umberto Scarfogliero, Michele Folgheraiter, Giuseppina Gini \\ Department of Electronic and Information , Politecnico di Milano \\ Piazza L. da Vinci, MILANO , I-20133, Italy32 \\ scarfogl@elet.polimi.it, folgher@elet.polimi.it, gini@elet.polimi.it
}

\begin{abstract}
This paper focuses on the study and design of an anthropomorphical light biped robot. The robot presents a total of twelve degree of freedom that will permit it to act a walk in a three dimensional space, right now tested only in simulation. Each joint resemble the functionalities of the human articulation and is moved by tendon connected with actuator located in the robot's pelvis. We implemented and tested an innovative actuator that permits to set the joint stiffness in real time maintaining a simple position control paradigm. The controller is able to estimate the external load measuring the spring deflection and demonstrated to be particularly robust respect to system uncertainties, such as inertia value changes. Comparing the resulting control law with existing models we found several similarities with the Equilibrium Point Theory.
\end{abstract}

Keywords: Humanoid Robotics; Biped; Joint Stiffness Control; Equilibrium Point Hypothesis

\section{Introduction}

The development of a humanoid robot usually requires relevant investments, comprehensive design and complex mathematical models. With LARP (Light Adaptive-Reactive biPed) we designed a simple and easy-to-reproduce biped, which could be at the same time cheap and efficient. Our aim was also to create a system that could represent a good model of human lower limbs. This in order to understand how the natural walking motion is achieved and how it can be implemented in a humanoid robot. For this reason, we adopted anthropomorphic feet, knees and a massdistribution similar to the human limbs. Several modern robots are designed to walk and behave like hu- mans [1] [2] but until now the efficiency of the human gait is still far from being reached.

In this sense, the work of McGeer [3] can be considered exemplar. His passive dynamic walker showed that without close position control, it is possible to perform a stable gait, considering the walking motion as a natural oscillation of a double pendulum; and this is actually how humans seem to walk [4] [5]. His results inspired many other works, such as the stability analysis on the compass model by Garcia et al. [6] and the physical implementation of several biped prototypes [7] [8] [9].

According to McGeer work, we designed an actuation system that can take advantage of the natural dynamic of the link. In addition, studing the results we got from our controller we found several similarities with the assumptions of the Equilibrium Point Theory. This is a widely debated theory, formulated in 1965 by A. Feldman [10] [11] [12], and still in evolution nowadays. In few words, this theory proposes that the segmental reflexes, together with the muscolo-skeletal system, behave like a spring. Movement is achieved just by moving the equilibrium position of that spring [13] [14] [15], and this is actually how our actuator, provided with visco-elastic elements, performs the movement. This similarity can be exploited to promote a further research in this sense, comparing the biped behaviour with human theories assumptions.

In section 2 the robot mechanical architecture is described, with particular attention to the knee, which present several similarities to the human articulation, and the foot, developed with two passive degrees of freedom. Section 3 reports the structure of our springdamper actuator and describes the control law we implemented. We also present the results we obtained running a preliminary simulation on the robot. Finally, the last section outlines the conclusions we can draw 
from our work and presents some future developments.

\section{The robot mechanical architecture}

\subsection{General outlines}

The robot we built (fig. 1) has 12 active degrees of freedom, is $90 \mathrm{~cm}$ tall and weights less than $5 \mathrm{~kg}$. It is entirely made by pieces cut out from a polycarbonate sheet. With the laser cutting technology, the practical realization of the robot is extremely simple. The material we used (polycarbonate) is a polymer that has a good strength-weigh ratio, can be widely deformed before breaking and is easy to be handled. Of course there are more performing materials, but we tried to build a robot that was not only light and simple, but also cheap.

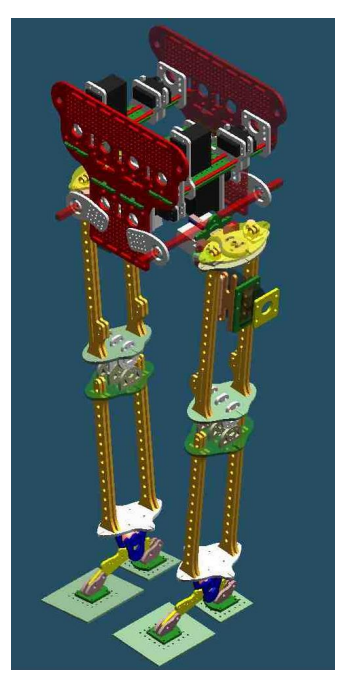

a.

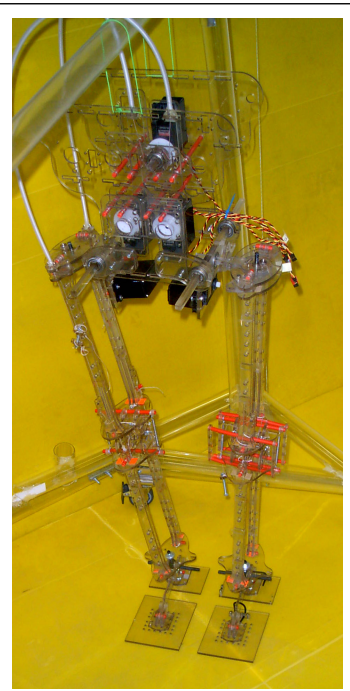

b.
Figure 1. (a) The 3D cad assembly of the robot. (b) The prototype itself, here with only one actuated leg.

Figure 2 shows the disposition of the twelve degrees of freedom in the robot. The range of motion of each joint is similar to that of humans during normal walking. Each foot has two passive degrees of freedom, this to ensure a reliable base during the whole stance phase. Joint torques are provided by servo motors disposed in the upper part of the robot. Thus we can obtain a very light leg, even with 6 actuated degrees of freedom. The transmission is performed by a simple system of cables and levers. The servo motors are equipped with a spring and a damper to permit the joint stiffness control.

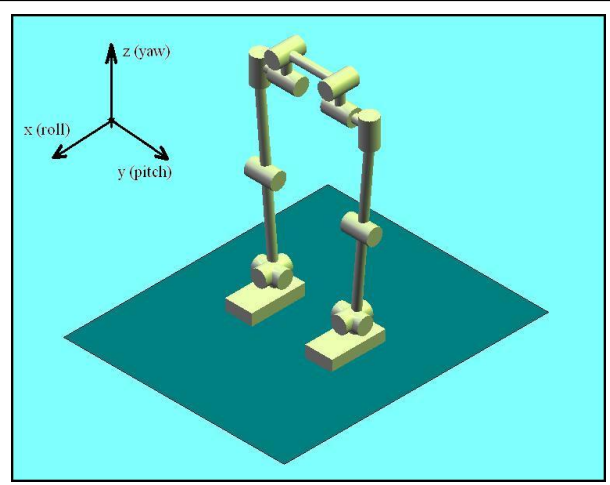

Figure 2. The disposition of the twelve degrees of freedom in the biped robot.

\subsection{The hip and the pelvis}

The hip joint has 3 degrees of freedom, disposed orthogonally (fig.3). The design is studied to limit the room needed by the joints, also considering that the motors are not directly applied to them.

The pelvis can host twelve big servo motors, equipped with a torsional spring and a damper. Ropes bring the motion to each joint of the robot. As some motors are included in the upper part of the thigh, there is also spare room for the actuation of an upper part of the robot.

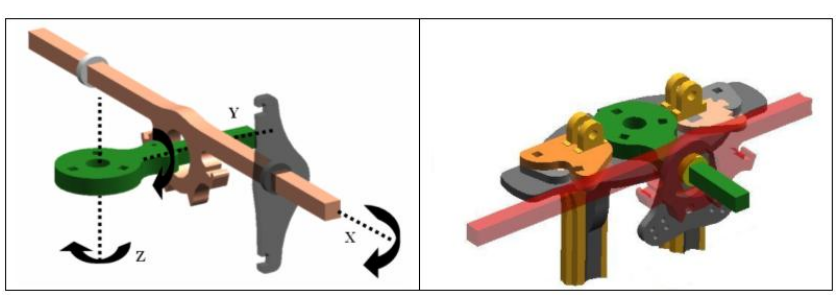

Figure 3. The structure of the hip and the pelvis. Here can be hosted up to twelve big servo motors. Noticeable is the fact that every part is derived by a planar sheet. 


\subsection{The knee}

Regarding the knee functions, the most obvious is lifting the shank for the foot clearance. In practice, if that was the only purpose of that joint, an hip articulation could make the job. Using stiff legs could actually simplify the motion and the robot structure (examples of this kind of robots go from the simple Fallis's toy [16] to the 3D biped robot of MIT LegLab). In practice, however, the knee has several important functions in the walking dynamic. Let's consider a robot with straight legs. To take a step the pelvis must be tilted to create foot clearance; this means a bigger energy consumption (as the pelvis is the heaviest part of the robot) and a reduced step length. This has a big influence on walking efficiency [17]. Another effect of straight knees would be that, during the step, the time of double support phase decreases, in behalf of the single support time. As the double support phase is the most stable position during walking, it is reasonable to tend to maximize it during the step. In this context, become fundamental the introduction of knee articulation.

About the actuation of this joint, we believe that it is not worth to use telescopic legs, as a pin joint permit to exploit the natural dynamic of the swinging motion, as in passive dynamic walkers [18] and is more human like. In order to minimize the energy consumption and the inertial forces of knee stretching, we designed this articulation to obtain the minimum friction and the maximum foot clearance with a small rotation. In addition, we had to keep it light, cheap and easy to produce and handle assembly.

This was achieved with the particular joint shown in figure 4 . This behave like a pin joint, but the center of rotation is not fixed: it rolls on the contact surface as the link rotates. The three parallel tendons leave only one degree of freedom in the X direction, and the resulting joint is really firm.

During the rotation, the tendons wrap on a surface or on the other, letting the link move without scratch, with a significantly reduced friction. Thanks to this joint, when the shank is swinging, the center of rotation moves upward and backward along the arc, and the foot clearance is increased by this motion. In this way, the shank rotation can be reduced significantly respect to a classical pin joint.

Regarding the radius of curvature of the two circular surfaces, we can optimize the rate of the two dimensions to have the maximum upward translation. As a matter of facts, if one surface (for example the upper one) has radius infinite or zero, the upward motion is null during the rotation. This means that there

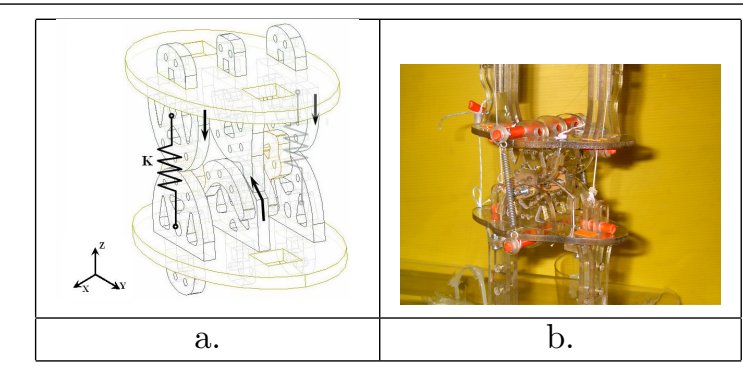

Figure 4. (a) The knee joint designed for our robot. The arrows show the way the tendons are wrapped. (b) The knee realized for the prototype

must be a finite rate value of the two radius that maximize the upward motion. In our biped, anyway, to keep the design simple, we adopted the same radius for the two surfaces.

In building the joint, we observed that the tension in tendons is critical for the robustness respect to torsional moment disturbances. To easily solve this issue, we preferred to add two elastic tendons rather than tightening the existing three.

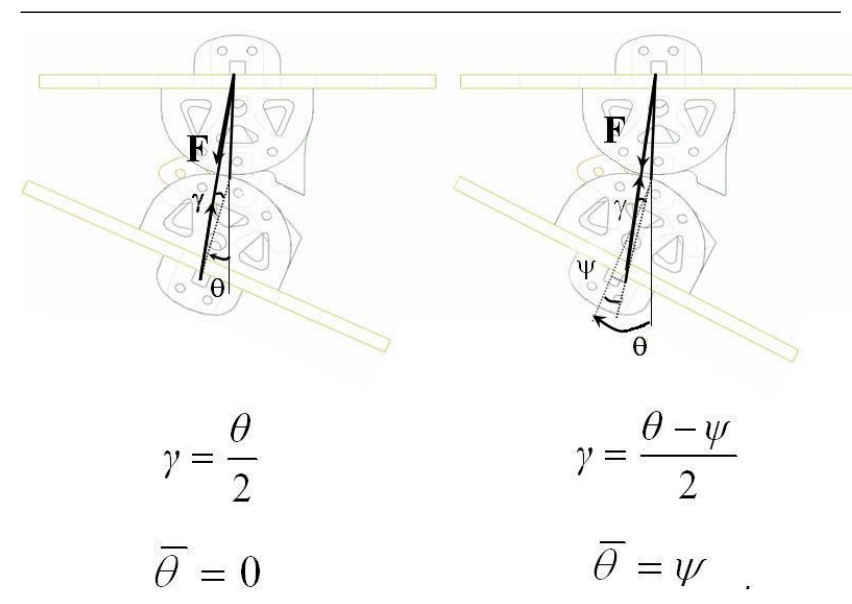

Figure 5. We can exploit the action of elastic tendons to impose a suited torque on the joint. In particular, it is possible to generate a position of instable equilibrium $(\theta=\bar{\theta})$ to favor knee banding or knee stretching.

For our design we decided to position the elastic tendons in a way that the force generated by the two springs helps the knee stretching and bending: we shifted the lower spring extremity forward and down- 
ward. As shown in fig.5, the position with $\theta=\bar{\theta}$ is an unstable equilibrium, and thanks to the springs action, the shank tends to rotate backward (knee bending) or forward (knee stretching).

\subsection{The foot and the ankle}

Another characteristic of the robot is the foot, designed in a way that really resemble the human one, not only in shape, but also in functions. The foot we used has two passive degrees of freedom, in the heel and in the toe (fig. 6), with spring-damper buffers to smooth rotation and absorb the impact. Also the sole helps cushioning during the ground contact; made in sobhortine, it can absorb the $95 \%$ of the impact force energy.

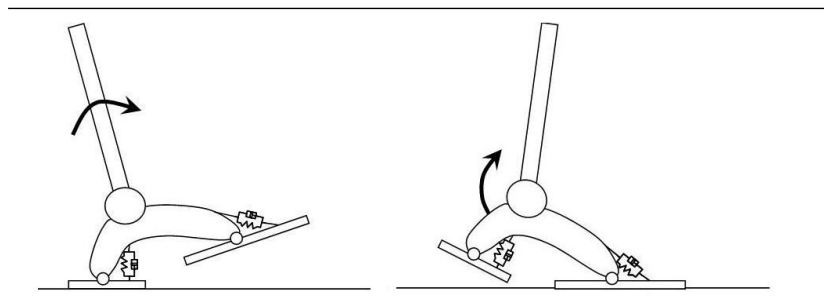

Figure 6 . The foot is composed by a main body and two passive joints. These have a fundamental function in walking stability and efficiency.

Alexander McN. [19], reporting the experiments of Ker et al. (1987), underlines that the foot behaves like an elastic body, returning about $78 \%$ of the energy in its elastic recoil. During running, the arc of the foot stores and returns $17 \%$ of the energy the body loses and regain at each footfall, while till the $35 \%$ of this energy is stored and returned by Achilles tendon.

For practical design, it was not possible to adopt an elastic material for the foot arc; thus, the whole buffering function was entrusted to the sole and to the two passive joints. In addition we inserted an artificial Achilles tendon between the heel and the arc of the foot.

These articulations in the foot have also a relevant influence on the kinematics and dynamics of the walking motion. As shown in figure 6, at heel-strike the foot body, and so the ankle position, are not constrained by the ground orientation. In this way the ankle joint is left free to rotate, keeping a firm base on which lean during the whole support phase. The same happens at toe-off, and the ankle can be moved forward and upward for knee-bending even keeping a stable ground contact. In this way, the double support time can be strongly increased respect to a classical flat foot and we have a firm support also during the toe-off. As Kuo and Donelan [20], [21] stated, this phase is fundamental in walking efficiency.

We can notice that during the support phase, the contact position moves from heel to toe. With our foot, the center of rotation $(c r)$ follows the same motion, while, with a flat foot, the $c r$ is constrained in the ankle joint. This means that, with our foot, the lever arm of the ground reaction force can be minimized, together with the energy consumption. As illustrated by Vaughan [22], joint torques, which represent a measure of the energy needed, can be approximated, in absence of large inertial contribution, with the moment of the contact force respect to the joint center. During the normal gait, as shown by Alexander [23], the line of action of the ground reaction force passes close to the hip, knee and ankle joints of the stance leg, minimizing in this way the energy consumption.

\section{The spring-damper actuation system with elastic reaction control}

\subsection{The spring-damper actuator}

The actuator is composed by a servo motor (we used big servos with $24 \mathrm{~kg}$ cm torque), a torsional spring and a damper. The resulting assembly is small, lightweight and simple, as we use a single torsional spring.

Using a spring between the motor and the joint let us have a precise force feedback simply measuring the deflection of the spring. The resulting actuator has a good shock tolerance; this is fundamental in walking, as impacts occur at every step. In addition, we can exploit the natural dynamic of the link storing energy in the spring. Similar actuators, with a DC motor and a spring, have been successfully used in biped robotics by Pratt et al. [24] and Yamaguchi and Takanishi [25].

The choice of the servos and the materials was made basically on cheap and off-the-shelf components. The main characteristic of this actuator is that the joint stiffness is not infinite, as it is in servo motors, and it can be changed in real time despite the constant stiffness of the spring. This has been achieved through a right choice of spring-damper characteristics and thanks to an intuitive control algorithm.

We must underline here that as joint stiffness we consider $k_{g}$

$$
k_{g}=\frac{M_{e}}{\varepsilon}
$$


where $M_{e}$ is the external load and $\varepsilon$ is the position error. A first prototype of our actuator was composed by two motors and two springs, working as agonist and antagonist muscles in humans. This let us to vary the joint stiffness even when no external load is acting, pre-tensioning the joint. With only one motor and one spring, the initial stiffness of the joint is fixed by the spring constant, this because the motor needs some time to tension the spring and counteract the external torque. Also, in this conditions, the presence of the damper in parallel to the spring permits to avoid high initial errors due to rapidly varying loads.

The damping factor can be chosen constant, at its critical value $(\xi=1)$

$$
\left\{\begin{array}{l}
w_{n}=\sqrt{k_{g} / I} \\
d=2 \xi w_{n} I
\end{array}\right.
$$

or can be varied during motion, in order to save motor torque and make the system faster. In the following paragraph we present the first option.

\subsection{The control algorithm}

The spring-damper actuator can be used in a torque control loop: the high-level controller assigns the torque to be delivered and, measuring the spring deflection, the low-level regulator makes the actuator perform the task. A way to assign joint torques is the Virtual Model Control developed by J. Pratt et al. [26]. In this approach, the controller set the actuator torques using the simulation results of a virtual mechanical component: like a spring, damper or any other mechanical device. In such a manner the robot can benefits of the component behavior without having it really.

In other classical approaches [27] the calculation of the joint torques is based instead on the dynamic model of the robot, that in many cases is complicated and imprecise. Indeed the biped robot can be formalized with a multi input multi output (MIMO) non linear system, that sometime presents also time variant dynamical behavior. In these conditions a classical PID (Proportional Integral Derivative) controller is not suitable and more complex control strategies are needed. On the other hand, if we apply only a simple position controller it remains to solve how to control the joint stiffness.

To solve these issues we developed a simple algorithm that can control the joint stiffness and position providing the worth torque without complex calculations. While a high-level controller assigns the trajectories, as in classical position control, the elastic low-level regulator permit to vary the joint stiffness in real time and make the actual position reach the reference one with a smooth motion.

In addition, we developed a more articulated algorithm, with acceleration and velocity feedback. This can provide an estimation of the external torque acting on the link, and modify the joint stiffness accordingly. These algorithms are described in detail in the next two sections.

3.2.1. The simplest control: position feedback The basic control algorithm is very simple and, as pointed out before, it is very close to a classical model of the Equilibrium Point hypothesis. It needs the reference position $\bar{\varphi}$ and the joint stiffness $k_{g}$ as inputs, and gives in output the motor position $\alpha_{0}$. The only state information needed is the actual joint position, that must be measured and fed back to the regulator. We may remind that the difference between the actual position and the motor one is covered by the spring deflection.

The control law is expressed by equation (2):

$$
\alpha_{0}=\frac{k_{g}}{k}(\bar{\varphi}-\varphi)+\varphi
$$

where $\mathrm{k}$ represent the spring stiffness, $\varphi$ and $\bar{\varphi}$ the actual and desired angular position respectively. The result is that a virtual spring with $k_{g}$ stiffness is acting between the reference angle and the actual position. We can notice that if $k_{g}=k$, we have $\alpha_{0}=\bar{\varphi}$, as the spring and joint stiffness coincide. On the other hand, if $k_{g}<k$ the motor rotation will be lower than the reference, as the spring stiffness is higher than the one required for the joint. Dually, if $k_{g}>k$ the motor has to rotate more to generate higher torques. Thus, the choice of $k_{g}$ and $\mathrm{k}$ can be made depending on the motor characteristics: $k_{g}>k$ attenuates the effects of a motor position error, while $k_{g}<k$ is suited when the motor limit is in the speed.

Regarding the other input, to avoid high initial acceleration $\bar{\varphi}$ should not be defined with steps, but, for example with second order functions with suited time constants. As a matter of facts, the finite joint stiffness betokens the presence of an error and one may define the time by which the desired position must be reached, accordingly with the joint stiffness. If this is very high, the error will be small, and the actual trajectory very close to the assigned one; this means that in presence of a step in $\bar{\varphi}$ high acceleration peaks can be generated. If the joint stiffness is small, one may expect relevant differences between the reference and actual trajectories, as the inertia and the damping oppose to fast movements. The static error $\epsilon$ depends anyway on the external load $\left(T_{\text {ext }}\right)$, as 


$$
\epsilon=\frac{T_{e x t}}{k_{g}}
$$

Equation (3) represents also a way to determine a proper joint stiffness, deciding the maximum error tolerance and estimating the external maximum load. Note that $k_{g}$ can be changed in real time, accordingly to the precision needed in critical phases of the motion.

To define the reference trajectory we used a step function filtered by a second order filter defined by a suited time constant $T$. In this way we can characterize the reference pattern with a single parameter.

To maintain the controller and the mechanical structure simple, the damping factor is set to a constant value that keep the system at the critical damping, as in equation (1).

We simulated the control of a simple 1-dof pendulum, and the results confirm the theoretical approach. In the simulation, gravity and varying external loads were included. Also friction was included to test the robustness of the algorithm.

The system parameters are:

$$
\begin{gathered}
m=1.2 \mathrm{~kg} ; \quad l=0.3 \mathrm{~m} ; I_{g}=7.35 \cdot 10^{-2} \mathrm{~kg} \mathrm{~m}^{2} ; \\
k=6 \mathrm{Nm} / \mathrm{rad} ; \mathrm{kg}=10 \mathrm{Nm} / \mathrm{rad}
\end{gathered}
$$

where $l$ is the distance between the center of mass and the joint axis.

Figure 7.a shows the behavior of the system: the commanded angle goes from zero to $0.3 \mathrm{rad}$ at $0.1 \mathrm{sec}$ and from $0.3 \mathrm{rad}$ to $-0.3 \mathrm{rad}$ at $1.2 \mathrm{sec}$ with a constant time $\mathrm{T}=0.08 \mathrm{~s}$. Here, only gravity is acting, but tests were made including variable external disturbances, which could mimic, for example, the inertia load of other moving links. The actual joint angle and the motor position are showed in the figure. With "static angle", we denote the position that the joint would have if the link inertia was zero and the damper was not present. To keep the figure clear the chosen stiffness is quite weak the error is about 0.1 rad only due to gravity. Looking at the motor position, we can notice that it is always opposite to the angle respect to the reference. This because here the spring stiffness is chosen lower than the joint stiffness. In this way the motor has to rotate more, but the system is less sensitive to motor position error. At about 1.4 sec., the motor rotation changes velocity due to servo maximum torque limit. In every simulation, also servo speed limitations were included.

Considering the resulting rotational acceleration, we can notice in fig.7.b that we have only two peaks, accel-

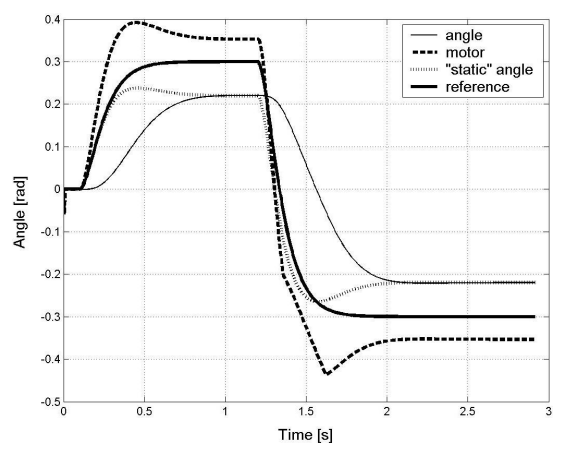

a.

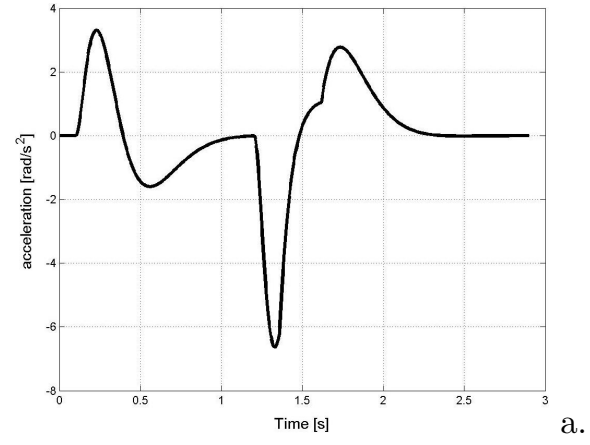

Figure 7. (a) The link rotation and the motor position referred to the commanded angle. We can see that the actual angle approaches the reference accordingly to the set stiffness and external load ("static" angle). (b) The acceleration pattern presents two peaks, characteristic of damped systems. The change at about $t=1.5$ $s$ is due to the limit on servo maximum torque.

eration and deceleration with no oscillation. This pattern, typical of damped systems, is particularly useful when it is needed to exploit the natural dynamics of multi-link systems. For instance, when starting a step, the acceleration of the thigh can be used to bend the knee, as in passive dynamic walkers [18] [9], or, before foot-fall, the deceleration of the swing motion can be exploited to straight the leg, as in passive lower-limb prosthesis.

To figure out the influence of rapidly external loads on the system behavior, we simulated a positioning task under step-varying external torque. Figure 8 shows the system under the action of an external load composed by a sinusoidal and constant action: at $0.1 \mathrm{~s}$ there is a positive step; at $1 \mathrm{~s}$ a negative one. Here the stiffness was highly increased, as a keep-position task was to be performed:

$$
k=10 \mathrm{Nm} / \mathrm{rad} ; \mathrm{kg}=50 \mathrm{Nm} / \mathrm{rad}
$$


Similar simulations have been run including a variable reference angle and friction at the joint.

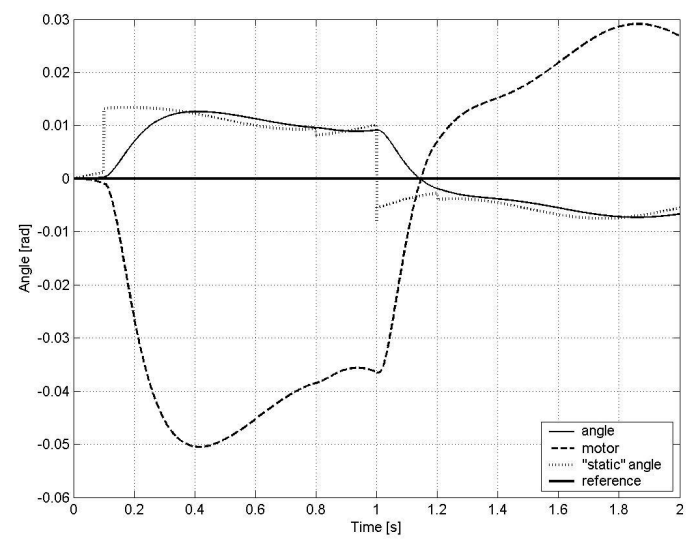

Figure 8. The system behavior under rapidlyvarying external torques. These can be seen in the "static angle" changing accordingly to the sinusoidal and step components of the load.

Thanks to this simple control law, we do not need to solve any inverse dynamic problem, but just decide the joint stiffness - using for example equation (3) and define the suited reference pattern. Different is the case, for instance, when, given a reference trajectory, we want to follow it controlling the motor torque; in this case, the external load plays a very important role, while, with the elastic control, we just need a rough estimate of it when the joint stiffness is fixed.

The following section describes a more complete algorithm that can automatically adapts joint stiffness to the external load in case that this dimensioning is not accurate. Regarding to the system, the only information needed is its inertia, or its average value for a multi-link system. In the next section It will be shown that the controller behaves robustly respect to inertia misestimation.

3.2.2. Force estimation through acceleration feedback Generally, in trajectory planning, not only the position is constrained, but also the velocity and acceleration must respect some limitations. This is especially important when we want to exploit the natural dynamic of the multi-body system; as we sketched above, the acceleration of the thigh can be used to bend the knee when starting the step [18] or to straight it before the foot-fall, as in passive leg prosthesis. Also velocity and accelera- tion limitations are needed where inertial loads, due to the movement of one part, can interfere with the motion of the rest of the robot; this is particularly relevant in bipedal walking.

To consider acceleration constrains, we included in our controller a sort of impedance control. By this term, we refer to the fact that the algorithm tracks the delivered torque and studies the resulting acceleration, creating a function relating these two quantities. In this way, we can create a simple dynamic model of a multi-body system without solving any inverse dynamic problem. The model can also get a good estimate of the external load acting on the joint; this can include the sole gravity or the interaction force with another links.

This can be obtained using, in the control loop, the equations:

$$
T_{e x t}^{i-1}=-k \cdot\left(\alpha_{0}^{i-1}-\varphi^{i-1}\right)+I \cdot \ddot{\varphi}^{i-1}+d \cdot \dot{\varphi}^{i-1}
$$

where $\mathrm{d}$ is the damping factor (see eq.1), $\alpha_{0}$ is obtained from eq. (2), $I$ is the inertia and $k$ an elastic constant. We can assume that between the instants $i-1$ and $i$ of the control loop the external load remains constant

$$
T_{e x t}^{i-1}=T_{e x t}^{i}
$$

Given the values of $\mathrm{k}, \mathrm{d}$,I, the position of the motor $\alpha_{0}$ and the estimation of $T_{\text {ext }}$, the acceleration can be foreseen as:

$$
A^{i}=\frac{k \cdot\left(\alpha_{0}^{i}-\varphi^{i}\right)+T_{e x t}^{i-1}-d \cdot \dot{\varphi}^{i}}{I}
$$

This is the way in which we implement a kind of impedance control: if the acceleration (system output) in the next step is different from the foreseen one, given the calculated $\alpha_{0}$ (system input), we infer that a different load is acting (system model has changed) and thus the motor position $\alpha_{0}$ is corrected accordingly. In some way this is also how we sample object properties in real word; for instance, to understand if a bin is empty or not we lift it and according to the resulting motion, we estimate the mass. The same we do to evaluate a spring stiffness, for example. In a positioning task, we make this sample-evaluation-correction every instant.

The simulations on a single joint brought to interesting results; with the same single joint as before:

$$
\begin{gathered}
m=1.2 \mathrm{~kg} ; l=0.3 \mathrm{~m} ; I_{g}=7.35 \cdot 10^{-2} \mathrm{kgm}^{2} ; \\
k=10 \mathrm{Nm} / \mathrm{rad} ; \mathrm{kg}=50 \mathrm{Nm} / \mathrm{rad}
\end{gathered}
$$


we could perform the motion evaluating the acceleration and the external load. In fig. 9 the results are shown with and without motor torque limitation. Here the external load is only the gravitational one. We can notice the effect of including motor torque limit, especially on the acceleration pattern.

As it is possible to see in fig. 9.c the characteristic is similar to the human electro-myographic activity, composed by there phases: acceleration-pause-deceleration [4], [15], and suitable for exploiting the natural dynamic of the links, i.e. in leg swinging as pointed out before.

From figures 9.e and .f we can also notice that the system perform a pretty good estimation of the external load acting on the link.

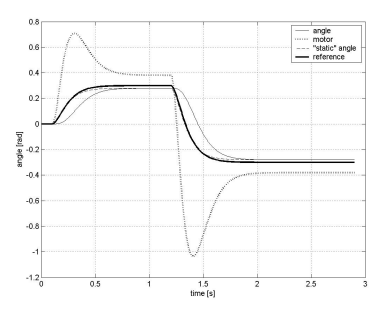

a

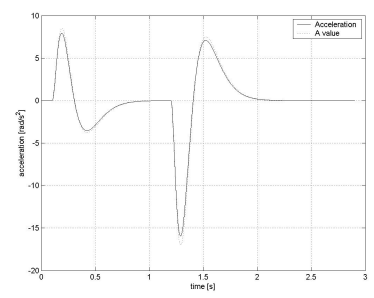

$\mathrm{c}$

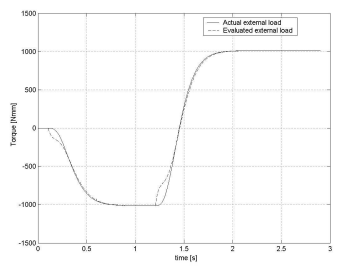

e

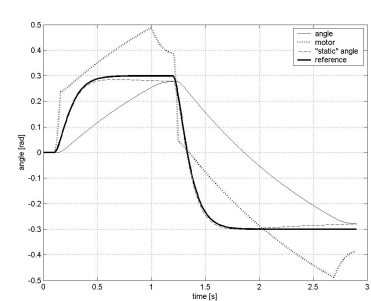

$\mathrm{b}$

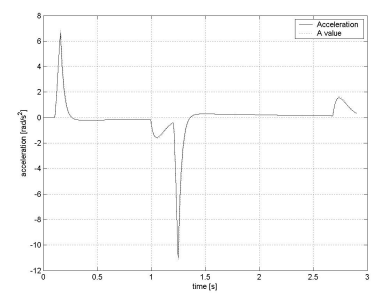

d

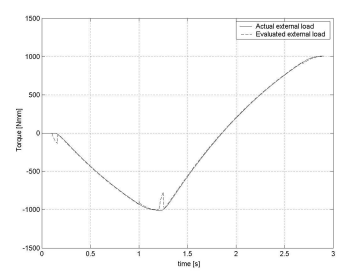

f
Figure 9. (a),(c),(e) show respectively the angles, the acceleration and its evaluation, $T_{\text {ext }}$ and its estimation when no motor torque limitation is considered. As we can see, the estimate is in good accordance with the real value. (b),(d),(f) show the same graph when a torque limitation is considered.

The controller can also perform a path monitoring on the acceleration; as a matter of facts, if the joint stiffness we imposed is, for example, too high for the load applied or the reference angle changes too quickly, the controller decrease the joint stiffness during the motion to prevent too high accelerations. This is done simply using the calculated acceleration value for the incoming iteration (eq. 5). If with the imposed stiffness the acceleration $A^{i}$ is too high, the low-level controller modifies $k_{g}$, given by the high-level algorithm, in order to respect acceleration limits. In this very simple way, we can ensure that the real value of the acceleration is kept under its maximum value, even despite wrong high-level commands.
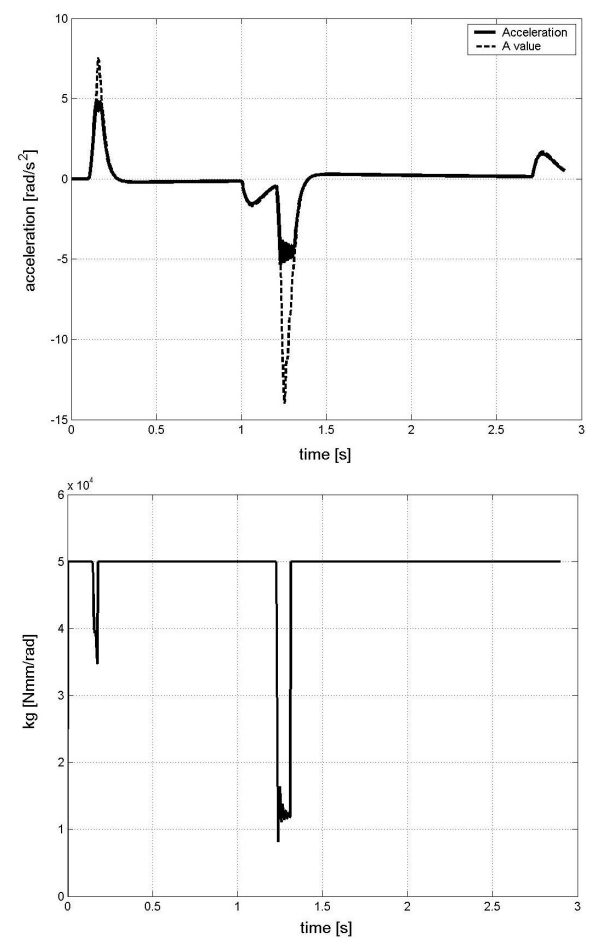

Figure 10. The algorithm can limit the acceleration acting on the joint stiffness without compromising the final positioning. This within few lines of calculations.

Setting the right joint stiffness can be guided by equation (3) or with a trial-and-error procedure. For example, a high-level learning algorithm could be used, not only to determine the $k_{g}$ value, but also the time constant of the reference trajectory. The choice of this two parameters as inputs for the low-level regulator is quite relevant: as a matter of facts, these two quantities can greatly influence the joint behavior, without hampering the final positioning. 
The only information the controller needs about the system is its inertia; in multi-link systems it can be approximated with a constant average value computed on all the links, or it can be calculated during the motion. In any case, the controller seems to be quite robust respect to inertia uncertainties, showing no relevant changes even for errors of about $30 \%$ (see fig. 11). As a matter of facts, the difference in inertia load is considered by the controller as an additional external torque. Regarding the damping, equation 1 can be rewritten as:

$$
d=2 \xi \cdot \sqrt{k_{g} I}
$$

This means that the damping factor is also proportional to the square root of inertia errors: while a too high inertia make the system over-damped, an underestimation can let the system have some oscillations. Anyway, the error in the inertia must be very high (such as 50\%) to see noticeable effect on the damping.

In the external torque estimation (fig. 11), we can notice the effect of wrong inertia input in the controller: for instance, if the real inertia value is higher, the controller acts as an additional external load is braking rotation during positive accelerations, as the real inertia is higher than what expected (see fig.f:Inertia). In this way, the system is "automatically compensated".

\section{The simulation on the robot}

The spring-reactive control has been implemented on our biped in a computer simulation. The robot model is shown in fig.12. As a first test, the robot had to preserve the equilibrium despite external disturbances. To run this test we implemented a simplified model; as a matter of facts, 6 dof are enough to perform the task; thus we only actuate two dof in the ankle (pitch and roll) and one in the hip (yaw) for each leg.

Figures 13 shows the external disturbances applied on the robot. The joint stiffness is set according to equation $(3)$, where $\varepsilon$ is the maximum error and $T_{\text {ext }}$ is the corresponding gravitational load. The value of inertia is calculated focusing on the resulting damping more than on the real value, that should be computed along the closed kinematic chain formed by the biped. Thus, for the ankle, we figure out the inertia of the robot considering the two feet coincident. Given the value of this inertia I, we evaluate the needed total damping factor d. As in the feet two dampers in parallel are present, we split the inertia so that the sum of the two dampers equal the total damping needed. Regarding the hip, we proceed in the same way, neglect-
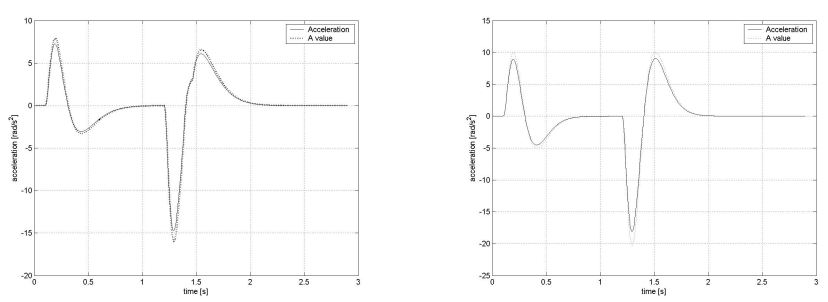

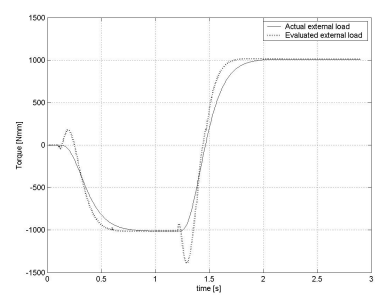

Overestimated Inertia

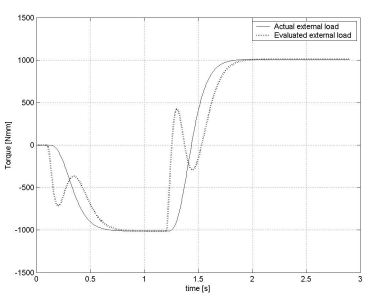

Underestimated Inertia
Figure 11. As we can see, an error of $30 \%$ in inertia value does not compromise the positioning; it is considered as an external additional load. If the computed inertia is lower than the real one, for example, when the system is accelerating, the algorithm interpret the too small acceleration (system response) as an external load that is braking the motion. On the other hand, when the computed inertia is higher than the real one, the system is over-accelerated, and a virtual additional positive torque is considered acting.

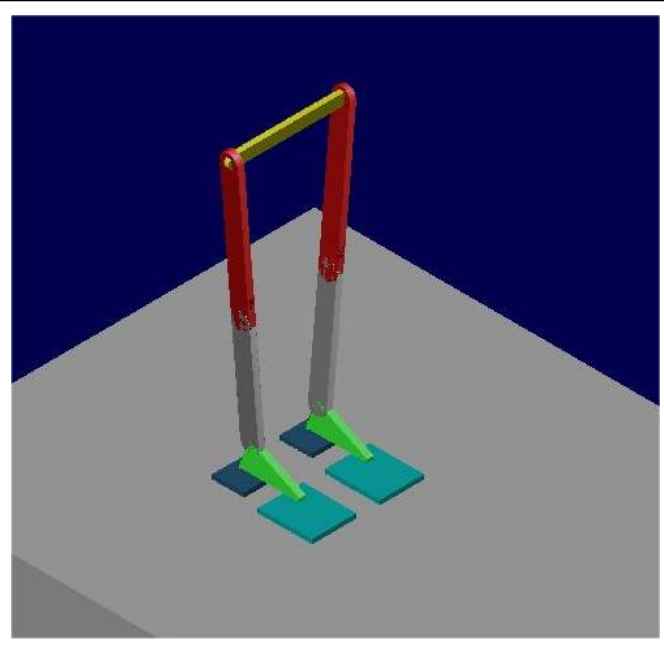

Figure 12. The robot model in the computer simulation. 
ing the leg beneath the joint for the inertia computation.

The results are shown in fig.14: we can notice that, as the disturbance is applied, a position error appears, as the actual angle differs from the reference position zero. The dotted line shows the motor rotation, that counteracts the disturbance and brings the joint back to the reference. In this way the robot is able to "react" to external loads, admitting a positioning error in order to preserve the whole balance.
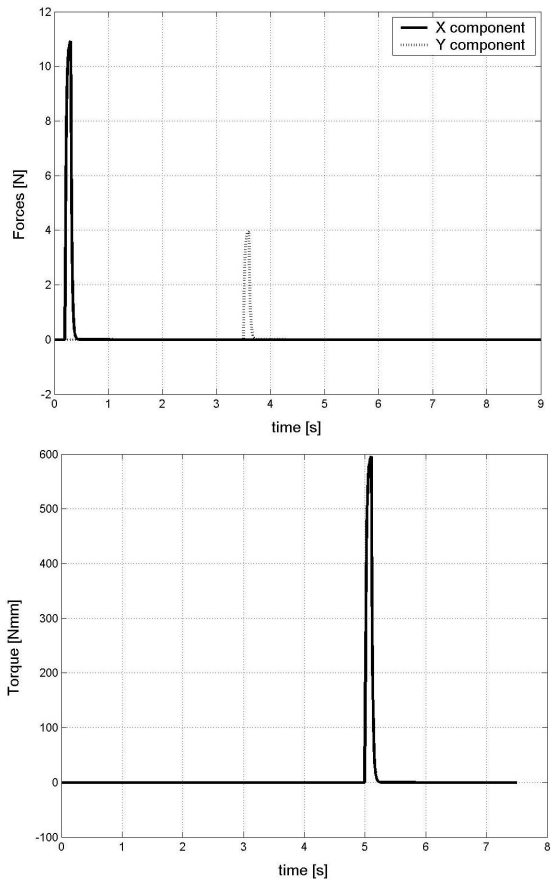

Figure 13. The external disturbances applied to the robot, forces and torque.

\section{Conclusions}

In this paper we described an innovative design for walking robot and an intuitive regulator for joint stiffness control. Our goal was to mimic the humans, in order to create not only a good biped, but also a structure that could model human lower limbs. For these reason, we developed an anthropomorphic knee joint and a foot with two passive dof. In addition, we tried to keep the mass distribution similar to the one of humans and to concentrate the mass in the upper part of the robot. Peculiar characteristic of our robot is that it is made up with pieces cut out automatically from
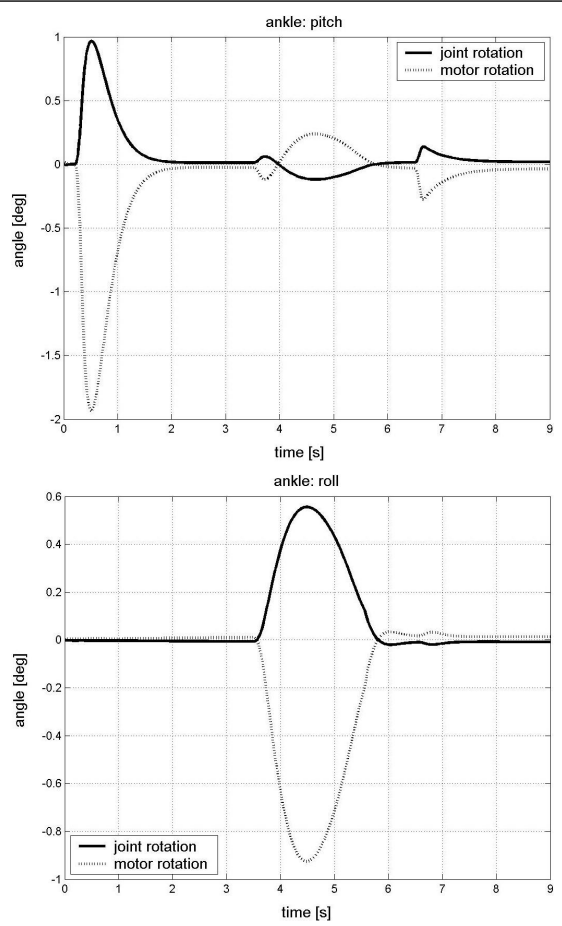

Figure 14. The angular position in the two degrees of freedom of the ankle: the disturbances are absorbed and the robot returns in its initial position.

a polycarbonate sheet. In this way, it is easy to adapt the robot to future changes, and it makes the biped easy to be reproduced. Regarding the actuation system, we designed a device equipped with a torsional spring and a damper. This allows to have a good shock tolerance and to estimate the external load measuring the spring deflection. Also, a method was developed to preserve the possibility of position control even with variable joint stiffness. This aspect is fundamental in biped robotics, not only to exploit the natural dynamics of the legs, but also to face with impacts occurring at every step. In this context we implemented a sort of impedance control that let the low-level regulator modify the assigned stiffness. Doing so, for example, we can avoid high accelerations in real-time and obtain a good estimation of the external load. In addition, the regulator demonstrated to be particularly robust respect to system uncertainties, such as inertia values.

Comparing the resulting control law with existing models, we found several similarities with the Equilibrium Point Hypothesis. Deeper researches can be made in this sense, using the system we developed as a model 
and studying the influence of changes in the control parameters. A future perspective is to compare the elastic actuator to human muscles and find out whether this kind of actuator can be used as a model of the complex muscolo-skeletal system. Further work can investigate the damper influence on the motion. In our simulations, to avoid oscillations along the assigned angle, the damping factor was fixed at the critical value. The drawback of this choice is that a relevant part of the motor torque is absorbed by the damper even when no external load is acting.

Thus, an alternative way is to choose the damping factor as an additional input parameter, to be controlled during the motion. According to the external load, the regulator could assign the damping needed to avoid oscillations and perform the right movement.

\section{References}

[1] Hashimoto S. at al. Humanoid robots in waseda university hadaly 2 and wabian. Autonomous Robots, $12: 25-38$, 2002.

[2] Haikawa Y. Takenaka T. Hirai K., Hirose M. The development of honda humanoid robot. IEEE International Conference on Robotics and Automation, pages 1321-1326, 1998.

[3] McGeer T. Passive dynamic walking. The International Journal of Robotics Research, 9(2):62-82, 1990.

[4] Kiriazov P. Humanoid robots: How to achieve humanlike motion. Journal of Biomechanics, (24):21-35, 1991.

[5] Hong D. Almeida G. Corcos D. Gottlieb G., Song Q. Coordinating movement at two joints:a principle of linear covariance. Neurophysiology, 75(5):1760-1764, 1996.

[6] Ruina A. Coleman M.J. Garcia M., Chatterje A. The simplest walking model: Stability, complexity and scaling. ASME Journal of Biomechanical Engineering Vol. 120 p.281-288, 120:281-288, 1998.

[7] Linde R. Q. vd. Wisse M., Schwab A. L. A 3d passive dynamic biped with yaw and roll compensation. Robotica, (19):275-284, 2001.

[8] Kuo A. D. Stabilization of lateral motion in passive dynamic walking. The International Journal of Robotics Research, 18(9):917-930, 1999.

[9] Ruina A. Collins S.H., Wisse M. A three dimensional passive-dynamic walking robot with two legs and knees. The International Journal of Robotics Research, 20(7):607-615, 2001.

[10] Feldman A.G. Asatryan, D.G. Functional tuning of the nervous system with control of movement or maintenance of a steady posture - i mechanographic analysis of the work of the joint or execution of a postural task. Biofizika, 10:837-846, 1965.

[11] Feldman A.G. Functional tuning of the nervous system with control of movement or maintenance of a steady posture - ii controllable parameters of the muscle. Biofizika, 11:498-508, 1966.
[12] Feldman A.G. Functional tuning of the nervous system with control of movement or maintenance of a steady posture - iii mechanographic analysis of the work of the joint or execution of a postural task. Biofizika, 11:667675, 1966.

[13] Gottlieb G.L. Latash, M.L. An equilibrium-point model for fast single-joint movement. similarity of single-joint isometric and isotonic descending commands. Journal of Motor Behavior, 23:163-191, 1991.

[14] J. McIntyre and E. Bizzi. Servo hypotheses for biological control of movement. Journal of Motor Behavior, 25(3):193-202, 1993.

[15] Corcos D.M. Agarwal G.C. Gottlieb, G.L. Strategies for the control of single mechanical degree of freedom voluntary movements. Behavioral and Brain Sciences, 12(2):189-210, 1989.

[16] Fallis G.T. Walking toy. U.S. Patent No.376588, 1888.

[17] Helm F. van der Koopman, B. and Veltink P. Lecture notes and textbook of the course 'Human motion control'. University of Twente, Enschede; University of Technology, Delft, 2001.

[18] McGeer T. Passive walking with knees. IEEE International Conference on Robotics and Automation, 2:1640$1645,1990$.

[19] Alexander R. McN. Energetics and optimization of human walking and running: The 2000 raymond pearl memorial lecture. American Journal of Numan Biology, 14:641-648, 2002.

[20] Kuo A. D. Energetics of actively powered locomotion using the simplest walking model. ASME Journal of Biomechanical Engineering, 124:281-288, 1998.

[21] Kuo D. A. Doneland J.M., Kram R. Simultaneus positive and negative external mechanical work in human walking. Journal of Biomechanics, 35:117-124, 2002.

[22] Vaughan C.L. Are joint torques the holy grail of human gait analysis? Human Movement Science, 15:423-443, 1996.

[23] Alexander R.McN. The Human Machine. Columbia University Press. New York, 1992.

[24] Williamson M.M. Pratt G.A. Series elastic actuators. IEEE International Conferences on Intelligent Robots and Systems, (1):399-406, 1995.

[25] Takanishi A. Yamaguchi J. Design of biped walking robot having antagonistic driven joint using nonlinear spring mechanism. IROS 97, pages 251-259, 1997.

[26] Torres A. Dilworth P. Pratt G. Pratt J., Chew C.M. Virtual model control: An intuitive approach for bipedal locomotion. The International Journal of Robotics Research, 20(2):129-143, 2001.

[27] Kwek L. C., Wong E. K., Loo C. K., and Rao M. V. C. Application of active force control and iterative learning in a 5-link biped robot. Journal of Intelligent and Robotic Systems, 37(2):143-162, 2003. 